Aus der Universitäts-Augenklinik Basel

Vorsteher: Prof. F. RinteLeN

\title{
Die Ophthalmologie in Basel zur Zeit des Barocks
}

\author{
Von F. Rintelen
}

Wie es schwer fällt, eine eindeutige Definition des Barockbegriffes zu geben, welche für die verschiedenen Manifestationen menschlichen Denkens, Fühlens und Wirkens charakteristisch sein könnte, so schwierig ist auch die zeitliche Abgrenzung dieser «Gegenregulationsperiode» - wie man sie mit einem medizinischen Begriff benennen könnte - nach Renaissance und Reformation. Wir werden nicht fehlgehen, sie grosso modo als dem 17. Jahrhundert zugehörig zu betrachten. Was für das Barocke in der Musik von Bach und Haendel, für Rubens und Bernini in der bildenden Kunst richtig sein mag, das wird nicht ohne weiteres auch für das Gebiet der inneren und äußeren Politik, für Philosophie und Naturwissenschaft Gültigkeit haben. Barock wirkt sich in den einzelnen Ländern und bei ihren Völkern auch ungleich aus, tritt keineswegs überall zu gleicher Zeit in Erscheinung. So können wir feststellen, daß der französische Barock des Grand Siècle Ludwigs XIV. - man hat ihn auch als die Zeit des Cartesianismus bezeichnet - nur schwer vergleichbar ist mit der deutschen in mancher Hinsicht repräsentativeren Barock-Kultur.

Die Naturwissenschaften in dieser Epoche betrachtend, spricht FrIEDELL von ihrem «Heldenzeitalter», und ähnliches mag, wenn auch in minderem Maß, für die Medizin gelten, die sich von der Präponderanz der statischen Anatomie des Vesal zur dynamischen Physiologie des Harvey und zur Hiatrochemie des Van Helmont fortentwickelt. Von wesentlichem Einfluß ist dabei die Philosophie, die ihrerseits durch naturwissenschaftliche Erkenntnisse befruchtet wird. BAGons: «Sine experientia nihil sufficienter sciri potest», und Descartes: "Cogito ergo sum», sind Maximen der barocken Epoche. Es ist weniger eine Zeit praktischen Fortschreitens als eine Epoche des Denkens und der Konzeption neuer Ideen, die sich auf die Erfahrungen des Versuches aufbauen. Es ist die Zeit, da Kepler seine Dioptrik, Scheiner sein Fundamentum Opticum schreibt, und LeeuwenноЕк ein brauchbares Mikroskop schafft, das die Sinneszellen der Netzhaut zu sehen erlaubt. Im barocken Zeitalter gibt es also keineswegs nur «wucherndes Unkraut», formalistische Bombastik und Exaltation, wie man 
sich das Denken und den Lebensstil dieser Epoche gerne, etwas primitivisierend, vorzustellen geneigt ist. In seiner Schrift über J. J. WePfer betont H.FIscher, «die Unterordnung der Erfahrung unter quantitativ bestimmte Gesetzmäßigkeiten haben gemeinsames Arbeiten in verschiedenen Ländern erlaubt, die menschliche Vernunft sei als einheitliche Kraft in den verschiedenen Kulturnationen zur Wirkung gekommen». Wir verstehen so auch das Aufkommen gelehrter Zeitschriften und die Gründung von Akademien. Barock ist Gegensätzlichkeit zwischen den aufkommenden induktiven naturwissenschaftlichen Methoden, welche die Medizin beherrschen, und dem Hang nach historischem Traditionalismus, Ambivalenz zwischen Rationalismus und pietistischen Bedürfnissen. Die Lehren des Hippokrates und Aristoteles, vor allem auch jene des Galen, leben in den neuen Lehren weiter. Im Theatrum Anatomicum läßt BAUHIN, der Basler Anatom, der keineswegs nur Überliefertes übernimmt, sondern viel Selbständiges hinzufügt, in den Randbemerkungen zu seinem Text die antiken Lehrer der Medizin zu Worte kommen, weit mehr als seine Lehrer Vesal und Plater.

In der Weitschweifigkeit und im Pathos der Darstellung, im Affektüberschwang der Zueignungen und Dankesbezeugungen, in der Art der Illustration kommt zumal in den deutschen Darstellungen das Barocke zur Geltung. Vielleicht ist es verständlich, daß die Menschen in deutschen Ländern durch den Dreißigjährigen Krieg und die Pest in Not und Elend gebracht, solchen Tröstungen zugänglicher und ihrer bedürftiger waren. In Frankreich dagegen, das Europa beherrscht, hat man diesen Überschwang weniger nötig, zudem liegt er den Franzosen a priori nicht. Wie sind etwa Descartes' Darstellungen jeder Bombastik abhold, wie bleiben sie klassisch streng, voller Klarheit im Denken und in der Sprache.

Die praktische Medizin, zumal die Chirurgie, die ja noch keine wissenschaftlich-medizinische Disziplin darstellte, hat in dieser Epoche wohl die geringsten Fortschritte und Erfolge aufzuweisen. Sie harrt der großen Systematiker des 18. Jahrhunderts, BoerhaAves in Leyden, Hoffmanns in Halle und SydenHams in London, welche die Errungenschaften der naturwissenschaftlichen Erkenntnisse des 17. Jahrhunderts zu greifbarer Frucht gebracht haben. Das 18. Jahrundert erst bringt auch die Synthese zwischen dem Wirken der praktischen Wundärzte und den gelehrten Herren von den Fakultäten, die der Barockperiode noch weitgehend fehlt.

Basel steht wie heute an einer sensiblen Berührungsstelle deutscher und französischer Kultur. Französische Klarheit Descartesscher Prägung und 
deutsche Phantasie wirken sich hier, vielleicht schwer zu synthetisieren und darum auch schwer zu resorbieren und zu assimilieren, gleichermaßen, wenn auch oft alternierend, aus. Diese Schwierigkeiten einer kritischen Sichtung und fruchtbaren Verwertung des gebotenen Stoffes waren vielleicht, ein Hauptgrund dafür, daß es an wirklich produktiven Leistungen und originellen Wegen gefehlt hat, obwohl Bauhin, Glaser, Harder und Th. ZwINGer II dazu durchaus geeignete Persönlichkeiten gewesen wären.

Wie sieht die Ophthalmologie des 17. Jahrhunderts aus, wenn wir ihre spezifischen und hervorstechenden Leistungen betrachten. Sie ist zweifellos beherrscht durch die Erkenntnisse der Physiologie, durch die Fortschritte physikalischen und mathematischen Denkens auf dem Gebiete der Optik. Porta, Kepler, Scheiner, Descartes und später Newton geben ihr den ersten Akzent. «Die Netzhaut und nicht die Linse ist der Ort des Sehens»,

\section{Vifio eft fentio affedx retiformis fpiritu vifivo plen $x$ : fi- $\checkmark e$, Videre, eft fentire affectam retiformem, quatenus affecla.}

Abb.1. Joannis KeplenI Dioptrice, Augustae Vindelicorum, David Frank, 1611

sagt uns Kepler. «Es entsteht auf der Retina ein umgekehrtes Netzhautbild », weist Scheiner nach, erklärt die Akkomodation richtig und gibt eine erste verständliche Optik des Auges heraus, ohne allerdings die höhere Mathematik, über die Kepler verfügt, zu gebrauchen, vielleicht auch, ohne sie gebrauchen zu können. $\mathrm{Da} ß$ seine Lehren für die damalige Zeit nicht leicht verständlich waren, hat der berühmte Jesuitenpater selbst empfunden: «Dico paucis multa, multis fortasse nimium!» In völliger Eindeutigkeit legt allerdings erst Descartes den Strahlengang im Auge dar und hält anschaulich im Bilde die Umkehr der Netzhautimago fest. Mariotte entdeckte 1666 den blinden Fleck!

Die praktische Augenheilkunde macht dagegen, wie HirschBerg sich ausdrückt, einen "traurigen Eindruck», sie befindet sich offenbar auf einem Tiefstand. Die Ärzte und die ordentlichen Chirurgen behandeln im allgemeinen die Augenkranken nicht, jedenfalls machen sie keine Staroperationen, die sie mehr oder weniger qualifizierten Wundärzten und Quacksalbern überlassen. Allerdings, das 17. Jahrhundert hat der Ophthalmologie nicht nur die Erkenntnis gebracht, daß die Netzhaut und nicht die Linse das eigentliche «Organum visus» ist, sondern auch erfaßt, daß der Sitz des grauen Stars nicht eine trübe Feuchtigkeit, ein Häutchen vor der Linse, 
vielmehr eine Trübung des Kristalls selbst darstellt. E.F.Quarre und H. LAsnier haben dies um 1640 in Paris auf Grund klinisch-chirurgischer Beobachtungen festgestellt. 1656 hat W. Rollfinck in Jena diese Auffassung anatomisch verifiziert. Allgemeinere Anerkennung fand die Lehre vom Sitz des grauen Stars aber erst im Anschluß an den berühmten Traité Brisseaus De la Cataracte et du Glaucoma, der 1709 in Paris erschien. Noch während über hundert Jahren hat es jedoch nicht an Gegnern gefehlt; vor allem ist der Engländer Woolhouse, ein praktisch geschickter, aber geldgieriger Scharlatan, der sich selbst als Ophthalmiatrum per Europam celeberrimum genannt hat, mit viel Gehässigkeit gegen die Starlehre Brisseaus aufgetreten. Die richtige Erkenntnis hat sich aber trotzdem durchgesetzt, nicht zuletzt durch den Einfluß von Lorenz Heister, den man als den Begründer der wissenschaftlichen Chirurgie in Deutschland bezeichnen kann; Heister empfiehlt übrigens den Chirurgen, sich der, wie er sagt, wunderbaren Operation anzunehmen, des Starstiches, der damals, bevor Daviel die eigentliche Starextraktion angab, in einer Reklination der trüben Linse in den Glaskörperraum bestand.

An brauchbaren Lehrbüchern der Augenheilkunde hat es im 17.Jahrhundert nicht gefehlt. Auf deutschem Sprachgebiet dominierte die schon 1583 erschienene Ophthalmoduleia, der Augendienst von Georg Bartisch, auf deutsch geschrieben, nicht das Werk eines Gelehrten, sondern eines Okulisten und Schnittarztes, das aber sowohl in seinen praktischen Angaben wie in der ethischen Grundhaltung auf beachtlicher Höhe steht : «Die Augen soll man hoch schätzen und sich den Star nicht auf dem Markt stechen lassen. Der Okulist soll fromm sein, Anatomie studiert haben, Wundarzt sein und dies Handwerk von Jugend auf gelernt haben. Er soll gesunde Augen haben, subtile Hände und Finger und auf beiden Händen hurtig sein, nicht geldgierig, nicht Trunkenbold, nicht ruhmredig; solcher Okulisten sind wenige zu finden », so schließt eine allgemeine Anleitung. Von Bartisch stammt die erste Angabe über die Enukleation, mit Bildern für das hiefür nötige Instrumentarium.

$\mathrm{Zu}$ den maßgeblichen Lehrbüchern der Augenheilkunde gehört vor allem das 1640 erschienene Handbuch der Medizin von Lazare Riviere (Medicina practica), wo den Affectibus oculorum - tam varii sunt atque multiplices, ut eos perspicere dificillimum sit - ein erstaunlich breiter Raum gewidmet ist. Das Buch muß auch für die Basler Ärzte von Nutzen gewesen sein, ist es doch 1663 vom damaligen Stadtarzt Bernhard Verzascha ins Lateinische übersetzt worden, von jenem merkwürdigen Manne, der es zum Mit- 

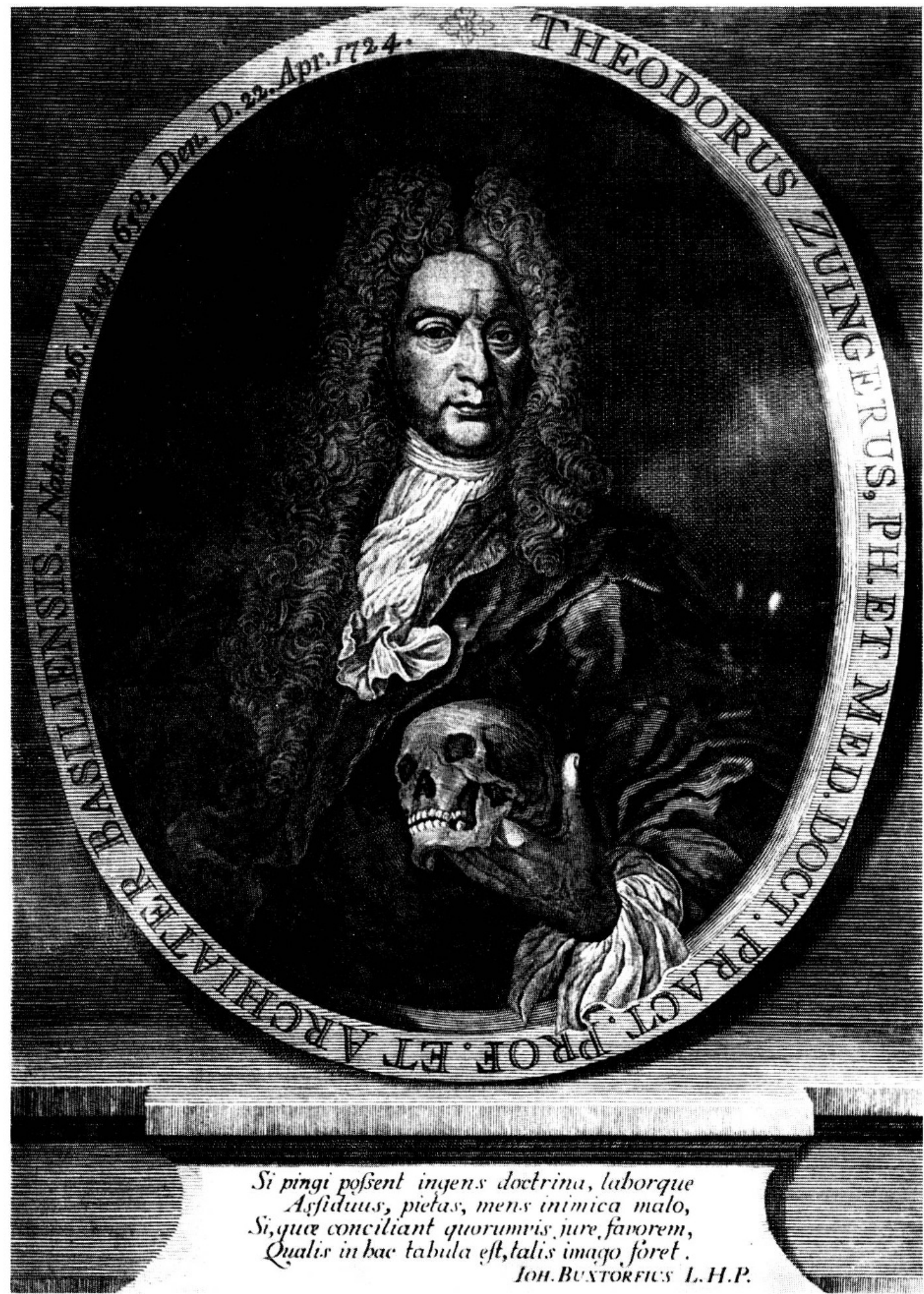

Abb. 2. Theodor Zwinger (der Jüngere), 1658-1724 
glied des Kleinen Rates brachte, mit der Fakultät aber, die ihn angeblich wegen eines Defektes in moribus nicht im Collegium medicum haben wollte, in heftigem Streite lag.

In der ersten Hälfte des 18. Jahrhunderts steht der Traité des Maladies de l'œil von Antoine Maitre-Jan obenan, 1707 in Troyes erschienen: ein Buch, das exaktes anatomisches und physiologisches Wissen mit großem klinischem Geschick, praktischer Erfahrung und erstaunlicher ethischer Haltung vereint. In der deutschen Übersetzung (Nürnberg 1725) sagt Maitre-Jan zur medikamentösen Therapie des Stars Dinge, die man auch heute noch aussprechen muß: "Ich schließe, daß man mit Arzteneyen keine Cataractam kurieren kann. So sich eine Cataracta entspinnet, soll man

De tout ce que deffus, je conclus qu'on ne peut guérir par les remedes les cataractes, quand même elles ne. feroient encore, que naiffantes ou non confirmées, \& qu'il eft tres difficile de les prévenir. Qu'ainfi, lorfqu'on a reconnu par les fignes diagnoftics ci-deffus expliquez, qu'une cataracte fe forme, on doit laiffer les malades en repos fans leur faire aucun remedes; à moins qu'il n'arrivât en même tems quelques autres maladies que l'on traiteroit fuivant les régles

Abb. 3. Antoine Maitre-Jan, Traité des Maladies de l'œil, Troyes, J. Le Febvre, 1707

die Patienten mit medizinieren ungeschoren lassen, man muntere sie auf, die Zeit geduldig zu erwarten, bis ihr Star zur Reifheit gelanget und zur Operation tüchtig geworden, und lasse sie einen glücklichen Ausgang ihres Zustandes hoffen, damit sie den Kummer vergessen.»

Praktische Wundärzte gelangten gelegentlich, falls sie eine gelehrte Vorbildung an den Fakultäten genossen hatten, wie Fabricius Hildanus, Stadtarzt von Bern und Meister der dortigen Schmiedenzunft, zu hohem Ansehen. Fabry verdanken wir die erste Magnetextraktion: Auf Anregung seiner Frau zieht er mit Hilfe des Magneteisensteins einen Eisensplitter aus der Hornhaut.

Wenn wir in Kürze einen Blick auf das Basel des 17. Jahrhunderts werfen, so stellen wir fest, daß es der Stadt schlecht geht. Selbst zwar nicht vom 
Kriege überzogen, leidet sie durch die ständige Gefährdung und durch die Verelendung der Nachbarschaft; im März 1638 sind 7000 Flüchtlinge in den Mauern; in der Nachbarschaft stehen die Bauern auf; die blutige Unterdrückung der Wirren führt zur Demütigung Liestals, die sich 200 Jahre später rächen sollte. In der Stadt kommt es zu einer kleinen Revolution gegen das Familien- und Zunftregiment. Gustav Steiner nennt dieses 91er Wesen «das traurigste Kapitel Basels Geschichte, das schlechtweg nur häßlich und niederträchtig sei». Bei dieser Gelegenheit wird der zünftige Chirurgus J. FATio, der in der Zunft zum Goldenen Sternen «vor ein Meuster in seinem Handwerk erkannt worden ", und der J.J.Harder, dem damaligen Inhaber des Anatomielehrstuhles an der Universität bei den Sektionen an die Hand gegangen und chirurgische Eingriffe an der Leiche demonstriert hatte, grundlos hingerichtet. Neben Not und Teuerung, neben politischen Unruhen wird die Stadt aber auch wiederholt von der Pest heimgesucht, welche die Bevölkerung in schauderhafter Weise dezimiert. Nach der Angabe von Felix Plater sterben allein im Jahre 16103681 Menschen an dieser unheimlichen Seuche bei einer Einwohnerzahl, die 1615 mit 16120 angegeben wird. Auch im 3. und 4. Jahrzehnt, sowie 1667/68 wütet die Seuche, und 1674/75 fordert das Fleckfieber unzählige Opfer.

So ist es verständlich, daß eine fruchtbare Entwicklung der Medizin in Forschung und Lehre in dieser Periode mit größten Schwierigkeiten verbunden ist. Äußerlich sehen wir das an den sinkenden Studentenzahlen. 1591 bis 1611 werden 441 Mediziner immatrikuliert, 1631 bis 1651 sind es noch 101 (A. BURGKHARDT).

Und trotzdem scheint es uns nicht richtig, wenn A. BURCKHARDT über diese Periode sagt, es habe an wirklich großen Persönlichkeiten gefehlt; wir gehen auch nicht mit ihm einig, daß von Ophthalmologie an der Fakultät damals nichts zu hören gewesen sei. Zwischen 1610 und 1725 haben wir immerhin 9 ophthalmologische Dissertationen gefunden, die zum Teil, besonders jene von G. Ch.Zollikofer, 1639 - Omnium praestantissimi sensus visus eiusque organi - sub auspice Felix Platers II, von beachtlichem Niveau sind.

Aus dem Gebiete der wissenschaftlichen Ophthalmologie seien nur zwei Fakten herausgegriffen. Caspar Bauhin, wir erwähnten es schon, hat in seinem Theatrum anatomicum, sicher auf Grund eigener Beobachtung, die Anatomie des Sehorgans in mancher Hinsicht erweitert. Wir erkennen das am besten auf Grund seiner Darstellung der Augenmuskeln, die wir mit den Abbildungen aus Vesal und Felix Plater vergleichen wollen. Die An- 


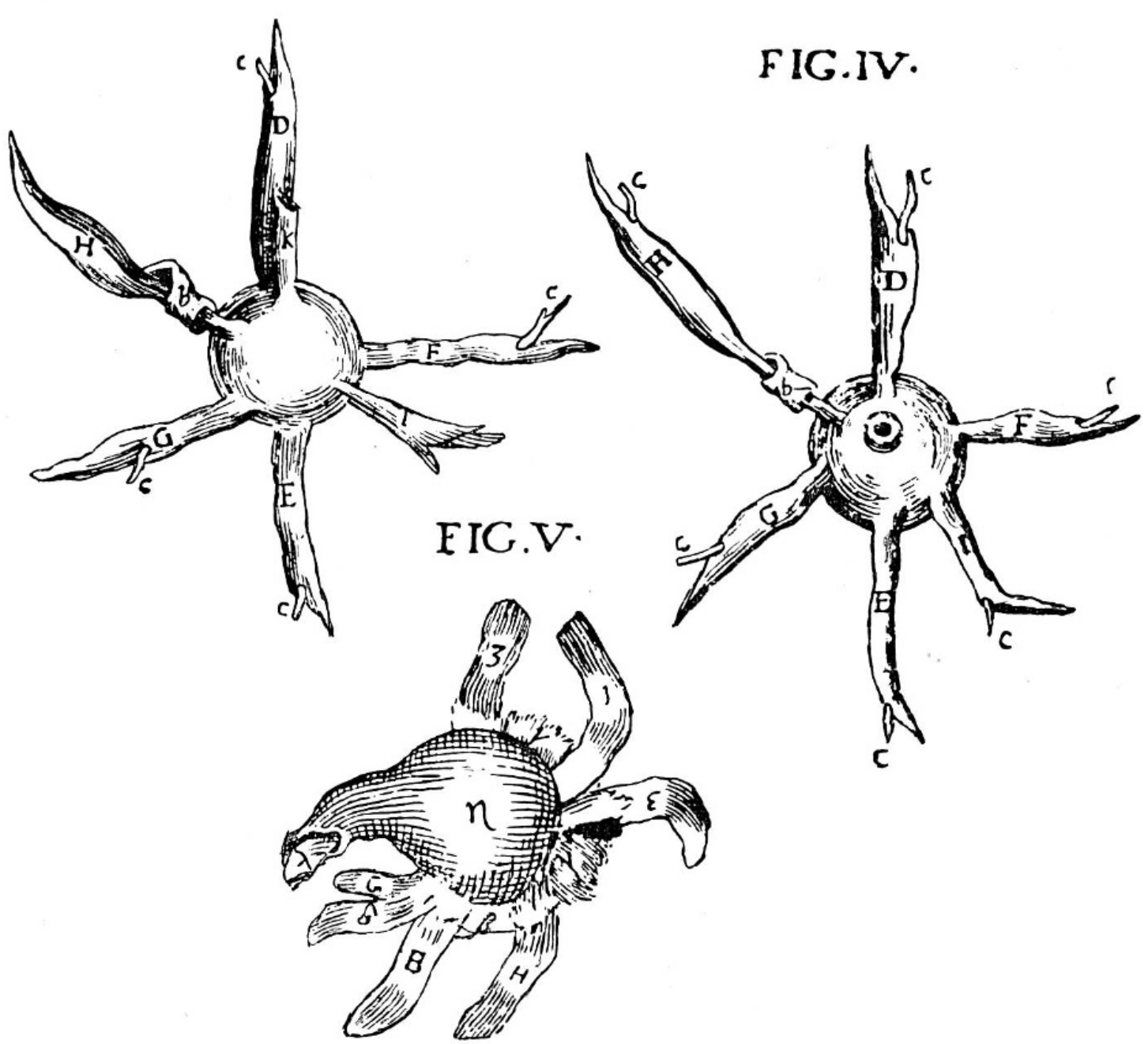

Abb. 4. Caspari Bauhini Theatrum Anatomicum, Francoforti, J.Th. De Bry, 1621

sätze der Muskeln sind bei Bauhin exakter und richtiger, der utopische siebente Augenmuskel F. Platers ist weggelassen. Bauhin widmet in seinem Theatrum übrigens nicht weniger als 10 Kapitel dem Sehorgan und seinen Adnexen und spricht am Schluß «De humorum oculi utilitate et visione» auch über physiologische Dinge.

1664 formuliert Doktor J.H.Glaser seine Theses opticae, eine Disputation zur Erlangung des Lehrstuhls für Mathematik. Ob sie sich, wie Burckhardt meint, auf Beobachtung gründen, scheint fraglich. Wir möchten vielmehr annehmen, daß Glaser sich wesentlich auf die Erkenntnisse von Kepler und Scheiner, vielleicht auch Descartes gestützt hat; einzelne seiner Thesen finden sich fast wörtlich in der Dioptrik von Kepler. Es ist aber diese Thesensammlung eine besonders klare, einfache, didaktisch geschickte 
Zusammenfassung des Wissens über die Optik des 17. Jahrhunderts. Halten wir drei der 29 Thesen fest:

«Das Sehen besteht darin, daß sichtbare Dinge in der Netzhaut aufgenommen und dank der dort vorhandenen Fähigkeit zur Erregung und Empfindung werden, darin auch, daß diese sichtbaren Dinge durch optische Empfindung (spiritus) zum Gesamtsinn überführt, zur Wahrnehmung werden » - eine erstaunliche intuitive Vorwegnahme wesentlicher Erkenntnisse unserer heutigen Sinnesphysiologie - Keplerschen Geistes.

«Das Bild wird auf der Netzhaut umgekehrt abgebildet, das kleine Bildchen, das wir beim Betrachten der Augen Anderer erblicken, ist nicht dasselbe, welches auf der Netzhaut abgebildet wird, sondern von ihm verschieden, es wird von den Strahlen, die vom Auge des Mitmenschen wie von einem Spiegel reflektiert werden, dem Auge des Beobachters eingeprägt »: das hatte Scheiner in seinen späteren Schriften begriffen.

Und endlich: «Daß ein Objekt einfach gesehen wird, erkennt man aus der Tatsache, daß die Sehlinien in beiden Augen gleich gelegen sind. So kommt es zur Abbildung des Bildes in gleicher Weise in beiden Augen. Infolgedessen nimmt das Bewußtsein (anima) durch diese zwei, sich ganz entsprechenden Bilder nur ein Objekt wahr» - eine klare Explikation des binokularen Einfachsehens.

Zum Mathematiklehrstuhl hat es trotz der Qualität der Thesen allerdings nicht gereicht. Glaser wird dafür Professor der griechischen Sprache. Wenn uns auch diese Potenz, gleichzeitig an drei verschiedenen Fakultäten ein Ordinariat versehen zu können, etwas mißtrauisch gegen die Qualität der möglichen Leistung stimmt, so staunen wir andererseits über die Universalität der Bildung damaliger Gelehrter.

Ausgesprochen barock-abrupt kommt nach der sachlichen Erörterung der optischen Thesen in den Corollaria, den Beigaben, der Satz «Hypothesis copernicana de Systhemate mundi rejicienda est. 1. Quia sensui repugnat, in primis quia S. Scripturae adversatur». Das ist wohl weniger, wie A. Burckhardt meint, eine Konzession an die Herren der theologischen Fakultät als die zeitgemäße Äußerung eines Gelehrten der Barockperiode.

$\mathrm{Zu}$ beträchtlichem Teil finden sich Glasers Thesen, wenn auch in etwas anderer, zum Teil ausführlicherer, zum Teil noch in wesentlich unklarerer Fassung, in seiner bekannten Schrift De Cerebro, einem ersten Leitfaden der Anatomie des zentralen Nervensystems.

Wie steht es nun mit den Behandlungsmethoden bei Augenleiden in Basel. Wir wissen darüber wenig. Immerhin steht uns ein recht interessantes Do- 
kument zur Verfügung, ein Brief aus dem Basler Staatsarchiv, den JoHANN Jаков Sснӧк, «Okulist, Stein- und Bruchschneider, des Rats und Kellermeister in Mülhausen », im April 1647 an die Fakultät gerichtet hat. Schön möchte von der Fakultät ein Zeugnis über seine Tätigkeit als Starstecher in der Stadt Basel haben. Da «ich nunmehr über die $20 \mathrm{Jahr}$ mein Kunst in loblicher Statt Basel vielfaltig exerziert, manche schöne Operation verrichtet, wobei von den Herren Medicis etliche Mal meinen Handgriffen beigewohnt haben, und ich jederzeit gethan, was einem ehrlichen Schnittarzt zusteht », bitte er um das Testimonium. Und die Fakultät scheint ihm durchaus gewogen. Der Dekan - Emanuel Stupanus - antwortet am 5. August 1647, daß er «in seine billige Begehr» eingewilliget. Herr Schön «sei seiner Kunst wohl erfahren, habe gute Wissenschaft erlanget und selbige zu Statt und Land Basel mehrmol exerziert und jederweil seine Erfahrung, Geschicklichkeit, unverdrossen Fleiß und große Treu erwiesen. Seine Fertigkeit in operationibus manualibus sei es wohl wert, ihme alle Fürderung, Hilf, Gunst und geneigten Willen würklich zu erzeigen.» Schön ist offenbar zünftiger, seßhafter Okulist in Mülhausen gewesen und hat Basel seine freundnachbarliche Hilfe als Starstecher zur Verfügung gestellt. In den Akten der Zunft zum Goldenen Sternen, wo die Wundärzte und Barbiere hingehören, sind, wie uns deren jetziger Meister, Dr. G.STEINER, berichtet, keine Einträge über die Examinierung von Okulisten, die zünftig werden wollen, oder auch von niedergelassenen Starstechern zu finden.

Wahrscheinlich sind manche Basler zu operativen Eingriffen am Auge ins Ausland gegangen, wofür wir allerdings in den sonst aufschlußreichen Leichenreden keine Hinweise gefunden haben.

Wie es damals um die nichtoperative, medikamentöse Therapie der Augenkrankheiten ausgesehen haben muß, können wir einer Schrift von Theodor Zwinger II entnehmen, der 1686 ein recht merkwürdiges Neues Arztneybuch: Der sichere und geschwinde Arzt bei Philipp Richter hat erscheinen lassen. Es steht auf einer ausgesprochen niedrigen, mit der sonstigen Qualität des Basler Anatomen kontrastierenden Stufe ärztlichen Wissens und Könnens. Es wendet sich bezeichnenderweise auch an alle Liebhaber der Artzneykunst, also an die Laien, die Curiosi, wie sie damals vielfach bezeichnet wurden. Wie primitiv, im Gegensatz etwa zu LAzARE Rivieres Ophthalmologie, Zwingers Darstellung ist, sei anhand zweier Beispiele illustriert. Bei der Augenblödigkeit heißt es: «Man siehst dreyerlei Augen, blaue, schwartze und graue, worunter die schwartzen die besten, benebend nicht häßlich, die blauen die schwächsten, aber lieblichsten, 


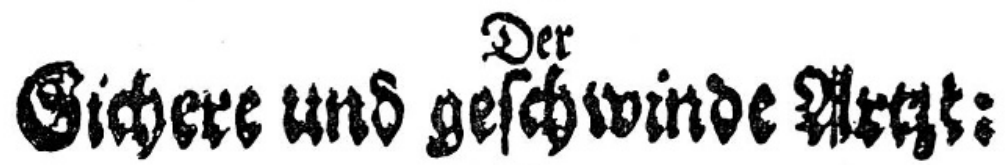

\author{
Ooer \\ Y)
}

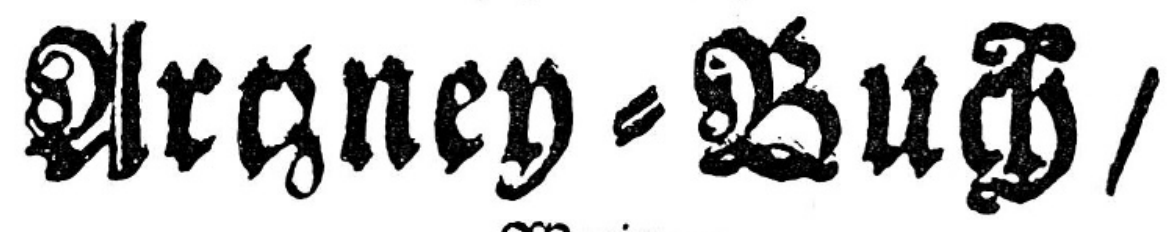

2Borinnell

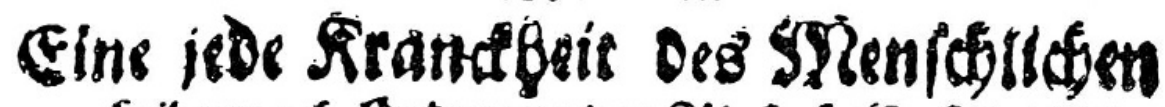
Scibs/nach Sronung oes 2llphabetho furrslich und grünolich befchrieben:

Ulilo

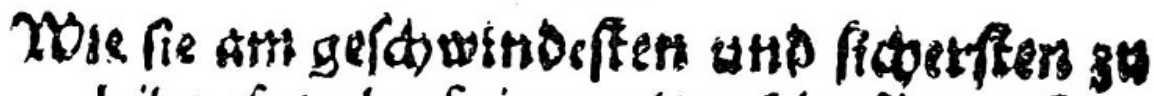
beilen / fo mol auf eigener als auch ber fürnembften

ঐerştenvielfaltigen Erfabrungan Den gelegt wirb.

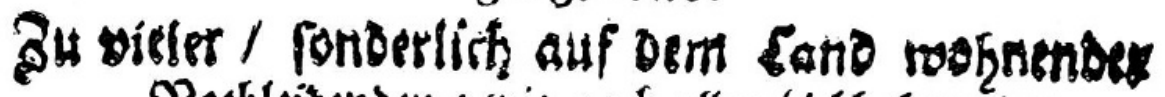
Dlotbleibenden/ roie auth aller siebfabern ber 2(rknely) funft mannigfaltigem

Nuken:

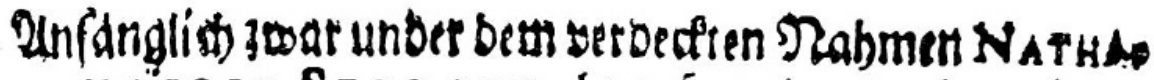
N A E I I S S F OR C I A beraubgegeben / anjeģo abes mit gettentem gleifife bermel)ret und verbefieret

\$ON

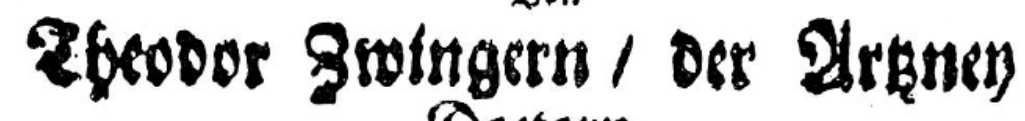
Doctorn.

\section{3afel /}

In Detlag Jobann- Philip Xiancrts

Dructes Jacob Berticje / I686,

Abb. 5 
die grauen die häßlichsten, benebend von mittelmäßiger Schärfe. Die großen Augen, welche die heutigen Frantzosen an ihrem Frauenzimmer sehr hochschätzen, sind insgemein schwach. Die kleinen aber schärffer und dauerhaffter. Sonsten kan das unauffhörliche Sauffen und Fressen, viel Studieren auf die Mahlzeiten die Lebensgeister schwächen, daß sie in geringer Menge nach den Augen fließen und einige zähe Feuchtigkeit darinnen hinderlassen, davon hernach das Gesicht verduncklet wird.» Beim Star soll man nach der Vorschrift von Herrn Verzascha, dem Stattarzt in Basel, folgendes Augenwasser verwenden: «Augentrostgeist, frische Baldrianwurzel, frische Schellkrautwurzel, frisch Eisenkraut und Rosmarin, Muskatnuß und Zimmet, frische Rauten, Fenchel und Äniß, frisch Augentrost; mische alles zusammen, vermache es, laß über Nacht stehen, destilliere es aus dem balneo Mariae und behalte es in einem wohlverwahrten Glas. Dieses Wasser kann man sowohl in- und auswendig gebrauchen.»

Ein anderes, weniger appetitliches Rezept lautet: «Wasser aus der Leber des Fisch Lamparte, Wasser aus Menschenkot, destilliert mit Zumischung der Gallen aus bedeutetem Fisch. Wenn aber der Star gleichwohl fortwachset, muß man verziehen, bis er in seiner Vollkommenheit, alsdann kan man ihne durch einen erfahrenen Meister ausstechen lassen.»

Der gleiche Mann, der so primitive Dinge publizieren konnte, wird 1694 Mitglied der Leopoldinischen Akademie der Naturforscher mit dem Gesellschaftsnamen Aristoteles, und man bemüht sich ernstlich, ihn für eine Professur nach Leyden zu gewinnen (A. BurcKHARdt, S. 196).

Noch wesentlich einfältiger ist der Guldene Artzney-Schatz mit Basler Hausmitteln vom Anonymus E. K. geschrieben, keinem Geringeren als dem Ordinarius für Physik und nachmaligen Inhaber der Cathedra theoretica, Emanuel König!

So finden wir auf dem Gebiet der Augenheilkunde in Basel im 17. und im ersten Viertel des 18. Jahrhunderts ein sehr eigenartiges, aber gerade für die barocke Kulturperiode typisches Gemisch: ausgesprochen gute Leistungen auf anatomischem und physiologischem Gebiet und wohl auch medizinisch-klinischem Erkennen, das die Herren der Fakultät lehrten und von ihren Dissertanten zum Ausdruck bringen ließen; wir dürfen annehmen, daß einige tüchtige Wundärzte als Starstecher am Werke waren. Und daneben blüht eine bedenklich primitive, pfuscherische therapeutische Praxis, würdig einer bissigen Molıèreschen Komödie. 